\title{
Magnetovolume and magnetocaloric effects in $\operatorname{Er}_{2} \mathrm{Fe}_{17}$
}

\author{
Pablo Álvarez-Alonso, ${ }^{1,2, *}$ Pedro Gorria, ${ }^{1, \dagger}$ Jesús A. Blanco, ${ }^{1, \dagger}$ Jorge Sánchez-Marcos, ${ }^{3}$ Gabriel J. Cuello, ${ }^{4}$ \\ Inés Puente-Orench, ${ }^{4,5}$ Jose Alberto Rodríguez-Velamazán, ${ }^{4,5}$ Gastón Garbarino, ${ }^{6}$ Imanol de Pedro, ${ }^{7}$ \\ Jesús Rodríguez Fernández, ${ }^{7}$ and José L. Sánchez Llamazares ${ }^{8}$ \\ ${ }^{1}$ Departamento de Física, Universidad de Oviedo, Calvo Sotelo s/n, 33007 Oviedo, Spain \\ ${ }^{2}$ Departamento de Electricidad y Electronica, Facultad de Ciencia y Tecnología, Universidad del País Vasco, \\ UPV/EHU, P.O. Box 644, 48080 Bilbao, Spain \\ ${ }^{3}$ Instituto de Ciencia de Materiales de Madrid, CSIC, Cantoblanco, 28049 Madrid, Spain \\ ${ }^{4}$ Institute Laue Langevin, 6 rue Jules Horowitz, 38042 Grenoble, France \\ ${ }^{5}$ Instituto de Ciencia de Materiales de Aragón, CSIC-Universidad de Zaragoza, 50009 Zaragoza, Spain \\ ${ }^{6}$ European Synchrotron Radiation Facility, BP 220, 6 rue Jules Horowitz, 38043 Grenoble Cedex, France \\ ${ }^{7}$ Departamento CITIMAC, Universidad de Cantabria, 39005 Santander, Spain \\ ${ }^{8}$ División de Materiales Avanzados, IPCyT, Camino a la presa San José 2055, 78216, San Luis Potosí, Mexico
}

(Received 1 February 2012; published 9 November 2012)

\begin{abstract}
Combining different experimental techniques, investigations in hexagonal $P 6_{3} / m m c \mathrm{Er}_{2} \mathrm{Fe}_{17}$ show remarkable magnetovolume anomalies below the Curie temperature, $T_{C}$. The spontaneous magnetostriction reaches $1.6 \times$ $10^{-2}$ at $5 \mathrm{~K}$ and falls to zero well above $T_{C}$, owing to short-range magnetic correlations. Moreover, $\mathrm{Er}_{2} \mathrm{Fe}_{17}$ exhibits direct and inverse magnetocaloric effects (MCE) with moderate isothermal magnetic entropy $\Delta S_{M}$, and adiabatic temperature $\Delta T_{\text {ad }}$ changes $\left[\Delta S_{M} \sim-4.7 \mathrm{~J}(\mathrm{kgK})^{-1}\right.$ and $\Delta T_{\mathrm{ad}} \sim 2.5 \mathrm{~K}$ near the $T_{C}$, and $\Delta S_{M} \sim 1.3 \mathrm{~J}(\mathrm{kgK})^{-1}$ and $\Delta T_{\mathrm{ad}} \sim-0.6 \mathrm{~K}$ at $40 \mathrm{~K}$ for $\Delta H=80 \mathrm{kOe}$, respectively, determined from magnetization measurements]. The existence of an inverse MCE seems to be related to a crystalline electric field-level crossover in the Er sublattice and the ferrimagnetic arrangement between the magnetic moments of the $\mathrm{Er}$ and $\mathrm{Fe}$ sublattice. The main trends found experimentally for the temperature dependence of $\Delta S_{M}$ and $\Delta T_{\text {ad }}$ as well as for the atomic magnetic moments are qualitatively well described considering a mean-field Hamiltonian that incorporates both crystalline electric field and exchange interactions. $\Delta S_{M}(T)$ and $\Delta T_{\text {ad }}(T)$ curves are essentially zero at $\sim 150 \mathrm{~K}$, the temperature where the transition from direct to inverse MCE occurs. A possible interplay between the MCE and the magnetovolume anomalies is also discussed.
\end{abstract}

DOI: 10.1103/PhysRevB.86.184411

PACS number(s): 75.80.+q, 75.30.Sg, 75.50.Bb, 61.05.F-

\section{INTRODUCTION}

In condensed matter physics, a rich diversity of magnetic phenomena, such as magnetovolume, magnetoresistance, and magnetocaloric effect (MCE), which are exhibited by rare-earth $(R) /$ transition-metal $(M)$ compounds (or $R-M$ ) makes them very promising for basic studies as well as for a large number of useful applications. ${ }^{1-4}$ For example, magnetic refrigeration, which could replace conventional gas compression technology, is based on MCE, and the search for new materials exhibiting this effect is a very attractive research topic. ${ }^{5,6}$ Magnetocaloric materials must display large adiabatic temperature $\left(\Delta T_{\mathrm{ad}}\right)$ and/or large isothermal magnetic entropy $\left(\Delta S_{M}\right)$ variations under an adiabatic magnetic field change. It is noteworthy that the shape and behavior of the $\Delta S_{M}$ or $\Delta S_{\text {ad }}$ curves can vary notoriously from one material to another, depending on the character of the magnetic phase transition. Three different shapes are usually found for $\Delta S_{M}(T)$ curves: (i) a sharp and narrow peak near the critical transition temperature, often associated with first-order magnetic phase transitions; $;, 8$ (ii) a caretlike shape characterized by a broad peak related to second-order phase transitions or crystalline electric field (CEF); 9,10 and (iii) a tablelike shape, i.e., a broad and flat peak of $\Delta S_{M}(T)$, linked to multiple sequential magnetic phase transitions. ${ }^{11,12}$

During recent decades, increasing attention has been paid to $R-M$ materials, from hard and soft binary intermetallic magnets to magnetic refrigeration. ${ }^{13-15}$ Furthermore, $R-M$ compounds (with $M=\mathrm{Fe}$ ) may have different crystal structures and magnetic behaviors, giving rise to diverse and unique properties associated with magnetic ordering and the existence of magnetovolume anomalies. ${ }^{2,16-19}$

Among them, the Fe-rich $R_{2} \mathrm{Fe}_{17}$ family of compounds is that with the highest $\mathrm{Fe}$-content within the $R$-Fe phase diagram. ${ }^{20} \quad R_{2} \mathrm{Fe}_{17}$ is a well-suited system to study $3 d$ and $4 f$ magnetism and the interplay between them, as the members of this family exhibit different ferro-, antiferro-, ferri-, helimagnetism, and fan magnetic structures as well as spin reorientation transitions, depending on the rare-earth element. ${ }^{21-25}$ These $R_{2} \mathrm{Fe}_{17}$ intermetallic compounds have attracted considerable attention since the discovery in the 1990's that interstitial $\mathrm{C}$ and $\mathrm{N}$ atoms provoke an increase of the Curie temperature $\left(T_{C}\right)$ and modify the character of the magnetocrystalline anisotropy, ${ }^{26,27}$ making some of them appropriate for use as permanent magnets. ${ }^{28}$ Nowadays, the existence of complex magnetic behaviors, including spin reorientations and first-order magnetic phase transitions, and the moderate MCE exhibited by some compounds have renewed the interest in these intermetallics. ${ }^{17,24,25,29-34}$

$R_{2} \mathrm{Fe}_{17}$ alloys crystallize in the rhombohedral space group $R \overline{3} m$ with a $\mathrm{Th}_{2} \mathrm{Zn}_{17}$-type crystal structure for light $R$ elements (less than a half-full $4 f$ shell), and in the hexagonal space group $P 6_{3} / m m c$ with a $\mathrm{Th}_{2} \mathrm{Ni}_{17}$-type crystal structure for heavy $R$ elements (more than a half-full $4 f$ shell). Those compounds with $R=\mathrm{Y}, \mathrm{Gd}$, and $\mathrm{Tb}$ can crystallize in either $\mathrm{Th}_{2} \mathrm{Zn}_{17}$ or $\mathrm{Th}_{2} \mathrm{Ni}_{17}$-type crystal structures, depending on the 
annealing process. ${ }^{35,36}$ In the $\mathrm{Th}_{2} \mathrm{Zn}_{17}$-type crystal structure, the crystallographic sites for the iron atoms are $6 \mathrm{c}, 9 \mathrm{~d}, 18 \mathrm{f}$, and $18 \mathrm{~h}$ in the Wyckoff notation and the unique $6 \mathrm{c}$ site for the rare earth atoms. For the $\mathrm{Th}_{2} \mathrm{Ni}_{17}$-type crystal structure, the $\mathrm{Fe}$ atoms occupy the $4 \mathrm{f}, 6 \mathrm{~g}, 12 \mathrm{j}$, and $12 \mathrm{k}$ sites, while the $R$ element is located at two nonequivalent positions, $2 \mathrm{~b}$ and $2 \mathrm{~d}$. In the latter, case, the structure can be a disordered variant in which the $R$ atoms also occupy the $2 \mathrm{c}$ sites and the iron atoms occupy the $4 \mathrm{e}$ sites. ${ }^{25,37-42}$ In addition, for $R_{2} \mathrm{Fe}_{17}$ alloys, the rare-earth magnetic anisotropy is rather weak and the contributions of the rare-earth atoms are not sufficiently strong to counteract the iron sublattice anisotropy at room temperature, which favors an easy plane magnetization. Recently, the body of experimental data on $R_{2} \mathrm{Fe}_{17}$ has been enriched with results from a moderate MCE. Values of $\Delta S_{M} \sim-6 \mathrm{~J}(\mathrm{kgK})^{-1}$ under a magnetic field variation of $\Delta H=50 \mathrm{kOe}$ are found around room temperature, for $R=\operatorname{Pr}$ or $\mathrm{Nd}$ compounds ${ }^{15,30,34}$ and pseudobinary alloys where two different $R$ elements are mixed. ${ }^{43,44}$ Since studies of the magnetocaloric response in $R_{2} \mathrm{Fe}_{17}$ are scarce, we have performed a thorough investigation into the heavy rare-earth Er, which is expected to show its larger MCE around room temperature.

In this paper, we report the crystal and magnetic structures of polycrystalline $\mathrm{Er}_{2} \mathrm{Fe}_{17}$ based on X-ray and neutron powder diffraction data. In addition, the MCE and magnetovolume anomalies exhibited by this alloy were studied by $\mathrm{x}$-ray powder diffraction under high pressure, dc magnetization, and specific-heat measurements. This article is organized as follows. Section II contains details concerning experimental methods and analysis procedures. Section III is devoted to give information on the Hamiltonian and the main interactions considered in $\mathrm{Er}_{2} \mathrm{Fe}_{17}$. The experimental and calculated results for the physical properties and their discussion are presented in Sec. IV, while Sec. V concludes the article.

\section{EXPERIMENTAL SECTION AND ANALYSIS DETAILS}

\section{A. Synthesis of $\mathrm{Er}_{2} \mathrm{Fe}_{17}$}

The starting materials for the preparation of $\mathrm{Er}_{2} \mathrm{Fe}_{17}$ as-cast pellets consist of pieces of commercial $\mathrm{Fe}$ and Er elements (Goodfellow $99.9 \%$ purity which, in the case of erbium, it is relative to the rare-earth content $)^{45}$ that were mixed in the nominal 2:17 molar ratio. Ingots of $4 \mathrm{~g}$ were prepared by the arc melting technique in an Ar atmosphere. The mixture was remelted three times to ensure their homogeneity. An excess of $5 \% \mathrm{Er}$ was added to compensate for evaporation losses during melting. Each specimen was wrapped in tantalum foil and sealed under vacuum in a quartz ampoule and then annealed for one week at $1373 \mathrm{~K}$. The annealing was followed by water quenching of the quartz ampoules immediately after removal from the furnace. Some of the resulting material was cut into pieces for magnetic and heat-capacity measurements. The rest was manually pulverized with an agate mortar and sieved $(106 \mu \mathrm{m})$ for powder diffraction experiments.

\section{B. Structural characterization of $\mathrm{Er}_{2} \mathrm{Fe}_{17}$}

The crystal structure and lattice parameters were studied by means of both x-ray (XRD) and neutron powder diffractions. Room-temperature XRD studies were performed in a high-resolution powder diffractometer (Seifert model XRD3000) operating in Bragg-Brentano geometry using $\mathrm{Cu}$ $K \alpha 1$ radiation $(\lambda=1.5406 \AA)$, with scans in $2 \theta$ taken between $2^{\circ}$ and $130^{\circ}$ with $\Delta 2 \theta$ steps of $0.025^{\circ}$ and counting times of $20 \mathrm{~s}$ per point. Two high-resolution neutron powder diffraction patterns, at $T=2$ and $320 \mathrm{~K}$, were collected on the D2B two-axis powder diffractometer (ILL, Grenoble, France) using a wavelength $\lambda$ of $1.59 \AA$. Also, 1-h diffraction patterns at selected temperatures between 5 and $325 \mathrm{~K}$ were collected for magnetic refinements on the D1B high-flux two-axis power diffractometer (ILL, Grenoble, France), with $\lambda=2.52 \AA$. Additionally, 5-min neutron diffraction patterns were collected on the same instrument in the temperature range from 5 to $850 \mathrm{~K}$ using a continuous and controlled heating rate of $1 \mathrm{~K} \mathrm{~min}^{-1}$ to determine the temperature dependence of the unit cell parameters. X-ray powder diffraction experiments under hydrostatic pressure were carried out on the ID27 beamline (ESRF, Grenoble, France) with $\lambda=0.3738 \AA$. $\mathrm{Er}_{2} \mathrm{Fe}_{17}$ powders were pressurized in a diamond anvil cell at room temperature. Neon was used as pressure-transmitting medium. A small ruby chip was placed in the sample chamber for pressure calibration. The collecting time of each pattern was $\sim 1$ min. Experimental raw data were reduced using the FIT2D program to obtain one-dimensional diffraction patterns. ${ }^{46}$ Analysis of the diffraction data, based on both Le Bail and Rietveld methods, were carried out with the FULLPROF suite package. ${ }^{47}$

\section{Magnetic characterization of $\mathrm{Er}_{2} \mathrm{Fe}_{17}$}

Magnetization measurements were carried out using a Quantum Design PPMS-9 T platform with a vibrating sample magnetometer option. The temperature dependence of the magnetization $M(T)$ was measured under three different values of the applied magnetic field, $H=50,1$, and $10 \mathrm{kOe}$. Isothermal magnetization versus applied magnetic field curves $M(H)$ were collected from 2 to $350 \mathrm{~K}$ with $T$ steps of 10 or $5 \mathrm{~K}$ for temperatures far from or close to $T_{C}$, respectively. For each $M(H)$ curve, the applied magnetic field was increased from 0 to $80 \mathrm{kOe}$ with steps of $0.5,1$, and $2 \mathrm{kOe}$. From $M(H, T)$ measurements, the isothermal magnetic entropy variation, $\Delta S_{M}(T, H)$, due to the change of the applied magnetic field from an initial value $H=0$ to a final value $H$ was calculated using the well-known Maxwell relation: ${ }^{15}$

$$
\Delta S_{M}(T, H)=\int_{0}^{H}\left[\frac{\partial M(t, h)}{\partial t}\right]_{t=T} d h .
$$

Calculations of $\Delta S_{M}$ at a given temperature were done by a numerical approximation of Eq. (1) replacing the partial derivative by finite differences and calculating the integral by numerical methods, using the same procedure as described in Ref. 15 . The specific-heat $C_{P}(T)$ was measured by a relaxation method in the 3-355 $\mathrm{K}$ temperature range under $H=0,20$, 50 , and $80 \mathrm{kOe}$ using a two- $\tau$ model analysis. The total entropy was calculated from $C_{P}(T, H)$ measurements:

$$
S_{\text {Total }}(T, H)=\int_{T_{1} \rightarrow 0}^{T} \frac{C_{P}(t, H)}{t} d t,
$$


Thus the magnetic entropy change was calculated as

$$
\Delta S_{M}(T, H)=S_{\text {Total }}(T, H)-S_{\text {Total }}(T, 0) .
$$

The adiabatic temperature change $\Delta T_{\mathrm{ad}}(T, H)$ was determined from the entropy-temperature diagram as the isentropic distance between the the zero-field entropy curve and that corresponding to $\mathrm{H.}^{48}$

\section{MEAN-FIELD THEORY}

The magnetic behavior of $\mathrm{Er}_{2} \mathrm{Fe}_{17}$ can be explained by a two-sublattice local-moment model. The temperature dependence of the $3 d(\mathrm{Fe})$ and $4 f\left(\mathrm{Er}^{3+}\right)$ magnetic moments, $M_{3 d}$ and $M_{4 f}$, respectively, was obtained in the framework of mean-field (MF) theory following a previously explained procedure. ${ }^{49}$ This allows us to approximate a many-body problem with a single-ion effective problem.

First, the magnetic interactions within the Fe sublattice were considered using a MF Heisenberg Hamiltonian (without considering the crystal-electric-field, CEF, interaction) with total spin angular momentum $S=1$ for all Fe atoms. $M_{3 d}$ can be determined numerically using the well-known relation $S B_{S}(y)=\langle\mathbf{S}\rangle$, where $B_{S}$ is the Brillouin function, and the magnetization $M_{3 d}$ can be written as $M_{3 d}=2 \mu_{B}\langle\mathbf{S}\rangle=$ $\mathbf{2} \mu_{\mathbf{B}} \mathbf{S B}_{\mathbf{S}}(\mathbf{y})$, with $y=\frac{2 \mu_{B} H_{3 d-3 d}}{k_{B} T}$, in which the molecular field is $H_{3 d-3 d}=\lambda_{3 d-3 d} M_{3 d}$. The value for the molecular-field constant $\lambda_{3 d-3 d}$ of $\sim 1700 \mathrm{kOe} \mu_{B}^{-1}$, was estimated in order to have a Curie temperature of $303 \mathrm{~K}$ for $\mathrm{Er}_{2} \mathrm{Fe}_{17}$, according to the experimental value obtained from the magnetization measurements (see below). Because the CEF interaction is neglected, simple expressions for the magnetic $\left(M_{3 d}\right)$ and thermodynamic quantities $\left(\Delta S_{3 d}\right.$ and $\left.\Delta T_{\mathrm{ad}}\right)$ can be determined. ${ }^{49}$ Second, the two Er sites in $\mathrm{Er}_{2} \mathrm{Fe}_{17}$ were considered as equivalent with respect to the value of their magnetic moments. The magnetism of $\mathrm{Er}^{3+}$ in $\mathrm{Er}_{2} \mathrm{Fe}_{17}$ can be understood from its Hund's rule ground state ${ }^{4} I_{15 / 2}$ with total angular momentum $J=15 / 2$.

Under the influence of a hexagonal CEF with local symmetry $D_{6 h}$ (z-quantization axis the $c$ axis), the Hamiltonian is given by

$$
\mathcal{H}_{\mathrm{CEF}}=B_{2}^{0} O_{2}^{0}+B_{4}^{0} O_{4}^{0}+B_{6}^{0} O_{6}^{0}+B_{6}^{6} O_{6}^{6},
$$

where the $O_{l}^{m}$ and $B_{l}^{m}$ are the Stevens operators and the CEF parameters, respectively. ${ }^{50}$ The sixteen-fold degenerate Hund's rule ground state splits into eight doublets, with CEF parameters $B_{2}^{0}=-1.65 \mathrm{~K}, B_{4}^{0}=-8.66 \times 10^{-3} \mathrm{~K}$, $B_{6}^{0}=-3.44 \times 10^{-5^{2}} \mathrm{~K}$, and $B_{6}^{6}=-1.65 \times 10^{-5} \mathrm{~K}$. These parameters were taken from the literature. ${ }^{51}$ As the ordered magnetic moments $M_{4 f}$ in $\mathrm{Er}_{2} \mathrm{Fe}_{17}$ are induced by $3 d-4 f$ exchange interactions, we combine the CEF Hamiltonian (4) with a mean-field Hamiltonian describing the $3 d-4 f$ exchange coupling (the $4 f-4 f$ exchange interaction is expected to be negligible compared to the $3 d-4 f$ exchange interaction):

$$
\mathcal{H}=\mathcal{H}_{\mathrm{CEF}}-H_{3 d-4 f} M_{4 f},
$$

where the molecular field $H_{3 d-4 f}=\lambda_{3 d-4 f} M_{3 d}$ is due to the $3 d-4 f$ exchange interaction, with $\lambda_{3 d-4 f}=-560 \mathrm{kOe} \mu_{B}^{-1} \cdot{ }^{42}$ In $R-M$ intermetallic systems, it is well known that the indirect exchange interaction between the $3 d$ spins of the transition elements and the $4 f$ spins of the $R$ elements exhibits a parallel coupling for light $R$ elements, while for heavy $R$ elements, this coupling is antiparallel. ${ }^{13}$ We obtain the MF eigenvectors and eigenvalues of the Hamiltonian (5) for one $\mathrm{Er}^{3+}$ ion. At a certain temperature, we start by considering the value of $M_{3 d}$, determined above, and an initial value for $M_{4 f}$. These quantities are introduced in the MF Hamiltonian (5). Once we get all the eigenvectors and eigenvalues, the new $M_{4 f}$ is calculated. This process is repeated self-consistently until convergence is reached. After the Hamiltonian is solved, ${ }^{9,52}$ the eigenvalues and eigenstates are used to calculate the partition function from which the temperature dependence of $M_{4 f}$, $\Delta S_{M}$, and $\Delta T_{\text {ad }}$ were calculated using the expressions given in Ref. 49.

In addition, it is generally accepted that the interplay of the $R$ and $M$ sublattice magnetic anisotropies governs the overall anisotropy in $R-M$ compounds. According to crystal field theory of $R-M$ intermetallic compounds, a positive second-order Stevens coefficient $\alpha_{j}$ of the $\mathrm{Er}^{3+}$ ion, combined with a negative second-order crystal field coefficient $V_{2}^{0}$, results in a uniaxial anisotropy of the Er-sublattice, while the anisotropy of the Fe-sublattice is planar. ${ }^{13,22,53,54}$ However, the magnitude of the $B_{2}^{0}$ coefficient of the Er sublattice is quite small compared to that of the Fe sublattice. Thus the anisotropy of the Fe sublattice dominates over the entire temperature range below $T_{C}$, and, subsequently, the easy magnetization direction of $\mathrm{Er}_{2} \mathrm{Fe}_{17}$ lies on the basal plane of the crystal structure.

\section{RESULTS AND DISCUSSION}

\section{A. Magnetic properties of $\mathrm{Er}_{2} \mathrm{Fe}_{17}$}

The temperature dependence of the magnetization, $M(T)$ curves, for different values of the applied magnetic field, is shown in Fig. 1. From the $M(T)$ curve measured under low magnetic field of 50 Oe (see inset in Fig. 1), the Curie temperature is estimated to be $T_{C}=303 \pm 1 \mathrm{~K}$, in good agreement with previously reported values. ${ }^{55,56}$ In addition, the magnetization displays a broad maximum around $150 \mathrm{~K}$ for applied magnetic field values over $1 \mathrm{kOe}$, as is illustrated in

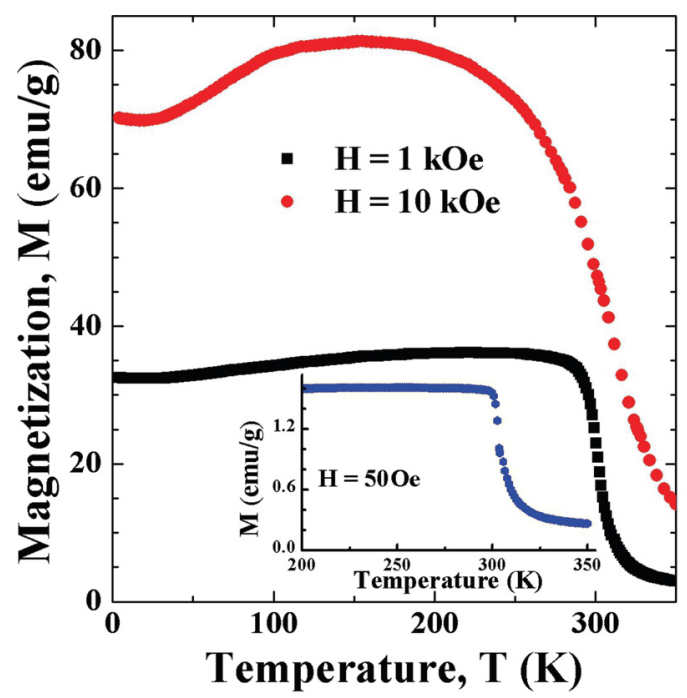

FIG. 1. (Color online) Temperature dependence of the magnetization, $M(T)$ for $\mathrm{Er}_{2} \mathrm{Fe}_{17}$ under $H=1$ and $10 \mathrm{kOe}$. (Inset) $M(T)$ curve at $H=50 \mathrm{Oe}$. 


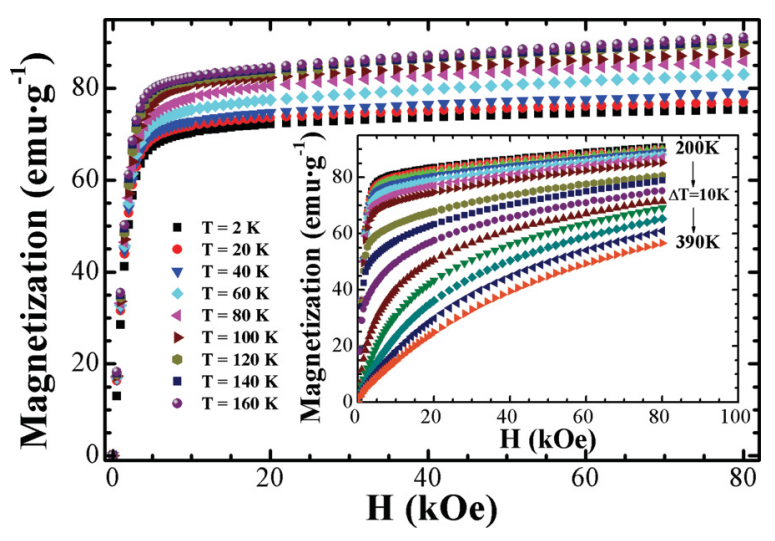

FIG. 2. (Color online) Applied magnetic field dependence of the magnetization in the temperature range where the $M(T)$ curve at $H=10 \mathrm{kOe}$ (see Fig. 1) increases with $T$. (Inset) $M(H)$ curves between 200 and $390 \mathrm{~K}$.

the $M(T)$ measured under $10 \mathrm{kOe}$ (see Fig. 1). In order to show the magnetic field dependence of the magnetization, $M(H)$, a number of $M(H)$ isotherms were measured (see Fig. 2). The saturation magnetization $M_{S}$ at $T=2 \mathrm{~K}$ was estimated from a fit of the $M(H)$ curve to the approach-to-saturation law: ${ }^{57}$

$$
M=M_{S}\left(1-\frac{b}{H^{2}}\right)+\chi_{0} H .
$$

The obtained $M_{S}$ value of $73 \pm 1 \mathrm{emu}^{-1} \mathrm{~g}\left(16 \pm 1 \mu_{B}\right)$

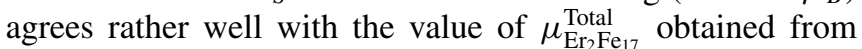
neutron powder diffraction (see below) and is close to the value given by Kuz'min et al. ${ }^{58}\left(82 \mathrm{emu} \mathrm{g} \mathrm{g}^{-1}\right.$ or $\left.18.8 \mu_{B}\right)$. At this temperature, the sample is approximately in the saturated state for $H>20 \mathrm{kOe}$. It is worth noting that the magnetic moment increases with temperature in the $2-150 \mathrm{~K}$ interval (see Fig. 2). The isothermal $M(H)$ curves change in such interval, especially between 40 and $80 \mathrm{~K}$, where an inverse MCE is found as we will discuss later. On the other hand, $M$ starts to progressively fall down above $200 \mathrm{~K}$ (see inset in Fig. 2), giving rise to a direct MCE. It is known that in $\mathrm{Er}_{2} \mathrm{Fe}_{17}$ single crystals there is a first-order field-induced spin-reorientation transition, ${ }^{58}$ which is reflected in an abrupt change of the $M(H)$ curves depending on the orientation of the single crystal with respect to the magnetic field. In this case, such a transition is not observed, mainly due to relatively low magnetic field values compared to the magnetic fields needed for such a transition ( $>300 \mathrm{kOe})$. For $T \sim T_{C}$, the magnetization at $H=80 \mathrm{kOe}$ is close to the value of the isotherm at $T=2 \mathrm{~K}$, which indicates that the magnetic field is able to increase the magnetization due to magnetovolume effects like in other well-known $\mathrm{Fe}$ invar alloys. ${ }^{25,59}$ For $T=390 \mathrm{~K}$, the magnetization at $H=80 \mathrm{kOe}$ is $\sim 60 \%$ of the magnetization at $T=160 \mathrm{~K}$.

\section{B. Heat capacity of $\mathrm{Er}_{2} \mathrm{Fe}_{17}$}

The temperature dependence of the heat capacity for $\mathrm{Er}_{2} \mathrm{Fe}_{17}$, measured under different magnetic fields up to $80 \mathrm{kOe}$, is shown in Fig. 3(a). The $\lambda$-type anomaly observed on the zero-field curve (maximum at $303 \pm 1 \mathrm{~K}$, inflection point at $305 \pm 1 \mathrm{~K}$ ) corresponds to the second-order magnetic phase transition from paramagnetic to ferrimagnetic state. Also

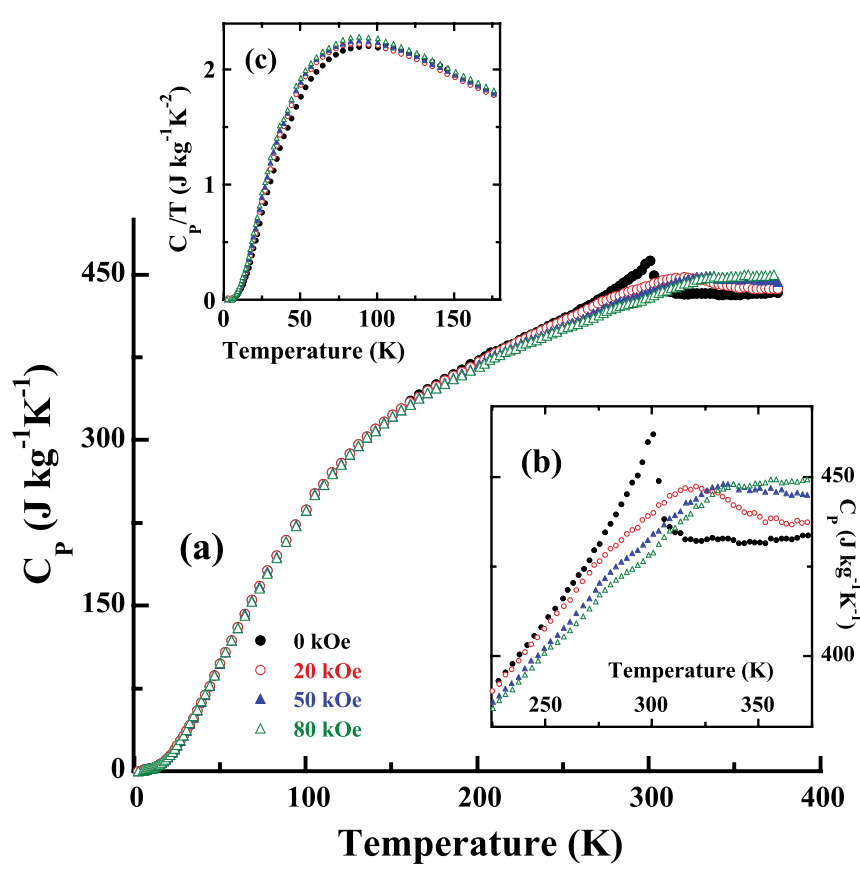

FIG. 3. (Color online) (a) Temperature dependence of the heat capacity for $\mathrm{Er}_{2} \mathrm{Fe}_{17}$ under different applied magnetic fields. (b) $C_{P} / T$ vs $T$ plot in the low-temperature range. (c) Detail of the heat capacity near the Curie temperature.

seen in Fig. 3(b) is the pronounced effect of the magnetic field on the $\lambda$-type anomaly (it is considerably broadened and shifted to higher temperature with increasing magnetic fields, which is characteristic of ferro- or ferrimagnetic systems). ${ }^{60}$ The temperature dependence of the heat capacity changes from 0 to $80 \mathrm{kOe}$ in the temperature range below $100 \mathrm{~K}$ [see $C_{P} / T$ plot in Fig. 3(c)]. This feature is relevant to understanding the appearance of an inverse magnetocaloric effect (see below).

\section{Crystal and magnetic structures of $\mathrm{Er}_{2} \mathrm{Fe}_{17}$}

Figure 4 depicts the high-resolution neutron powder diffraction patterns for $\mathrm{Er}_{2} \mathrm{Fe}_{17}$ collected on D2B at 2 and $320 \mathrm{~K}\left(T_{C} \sim 303 \mathrm{~K}\right)$. The diffraction pattern obtained for the paramagnetic state at $320 \mathrm{~K}$ can be indexed with Bragg reflections associated with the $\mathrm{Th}_{2} \mathrm{Ni}_{17}$-type crystal structure, which coincides with room temperature $\mathrm{x}$-ray diffraction data (not shown here). The cell parameters and the atomic coordinates after refinement are in good agreement with those previously reported. ${ }^{37,61}$ The structural parameters were obtained from the Rietveld analysis (see Table I). Labels of $\mathrm{Er}$ and $\mathrm{Fe}$ atomic coordinates correspond to those used in the International Tables for Crystallography. ${ }^{62}$ It is noteworthy that several refinements using the disordered $\mathrm{Th}_{2} \mathrm{Ni}_{17}$-type crystal structure (which is found in other hexagonal $R_{2} \mathrm{Fe}_{17}$ compounds) ${ }^{38,63,64}$ were carried out, but led to results with poor reliability factors and unphysical results.

The nonstructural parameters (scale factor, zero shift of the goniometer, and profile parameters for the pseudo-Voigt function) and the atomic coordinates obtained from the Rietveld refinement of the pattern collected at $320 \mathrm{~K}$ were used for the $2 \mathrm{~K}$ diffraction pattern, in order to reduce the number of variables in the fitting procedure. At $T=2 \mathrm{~K}$, 


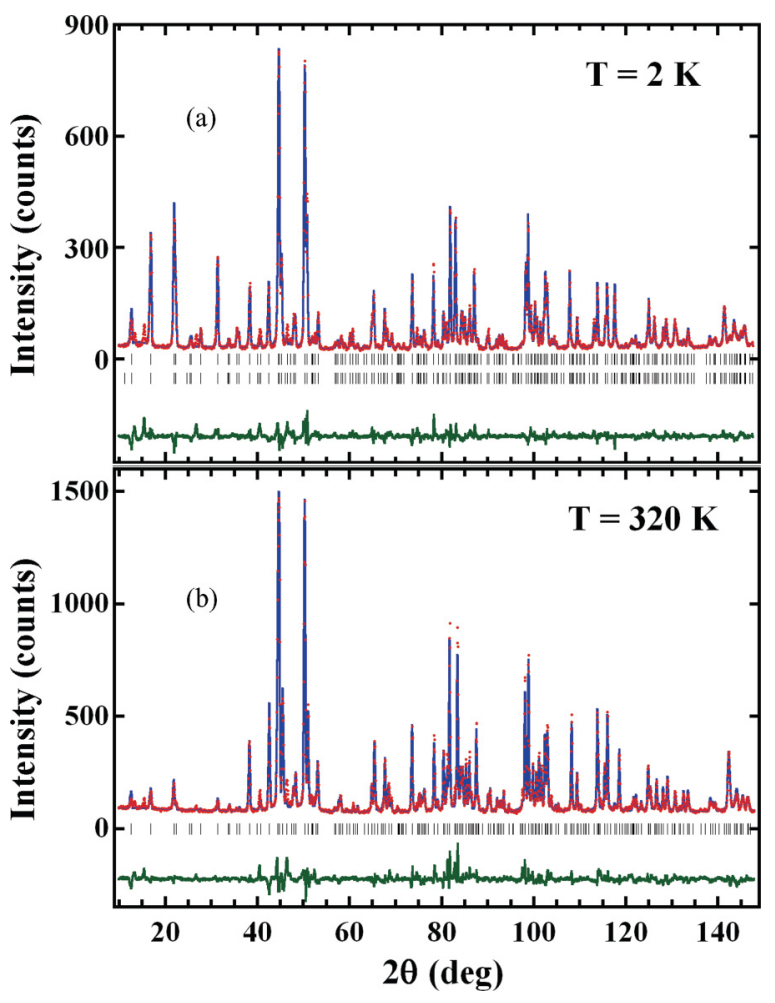

FIG. 4. (Color online) Observed (dots) and calculated (solid line) neutron powder diffraction patterns collected on D2B at (a) $T=2 \mathrm{~K}$ and (b) $T=320 \mathrm{~K}$ for $\mathrm{Er}_{2} \mathrm{Fe}_{17}$. The vertical bars indicate the position of the Bragg diffraction reflections; the upper (lower) row corresponds to the nuclear (magnetic) scattering. The observedcalculated difference is depicted at the bottom of each figure.

$\mathrm{Er}_{2} \mathrm{Fe}_{17}$ shows a long-range ordered magnetic state. In the $\mathrm{Fe}$ sublattice, the magnetic moments were refined using the $\mathrm{Fe}$ magnetic moments obtained from $\mathrm{Y}_{2} \mathrm{Fe}_{17}$ as starting values. Considering that the Er crystallographic sites $(2 \mathrm{~b}$ and $2 \mathrm{~d}$, see Table I) possess similar atomic environment due to their local symmetry, the Er magnetic moments were constrained each other (see Sec. III). All magnetic peaks were indexed with a propagation vector $\boldsymbol{k}=(0,0,0)$ referenced to the room temperature unit cell, indicating that the magnetic and nuclear unit cells are the same. The magnetic cell resulting from refinement is collinear ferrimagnetic with the magnetic moments lying in the basal plane, the Er-sublattice magnetic moments being antiparallel to those of the Fe sublattice. The value of the Er magnetic moment at $T=2 \mathrm{~K}$ is close to that of the maximum value predicted for the $\mathrm{Er}^{3+}$ free ion according to Hund's rules $\left(\mu_{\mathrm{Er}}=9 \mu_{B}\right)$. It is noteworthy that the components of the magnetic moments within the basal plane cannot be determined from a neutron powder diffraction experiment due to symmetry limitations. ${ }^{65}$

The temperature dependence of the magnetic moment at each nonequivalent atomic site for $\mathrm{Er}_{2} \mathrm{Fe}_{17}$ was calculated from the analysis of the powder diffraction patterns collected on D1B. As can be seen in Fig. 5(a), the temperature dependence of the magnetic moments for Er and Fe ions, $\mu_{\mathrm{Er}}$ and $\mu_{\mathrm{Fe}}$, are significantly different. The Er magnetic moment decreases monotonously, whereas the magnetic moments of the Fe atoms remain almost constant until $T \sim 200 \mathrm{~K}\left(T / T_{C} \sim 0.7\right)$, with an abrupt decrease near $T_{C}$. Figure 5(b) shows the total magnetic moment as a function of the reduced temperature $\left(T / T_{C}\right)$ for each sublattice, where the spontaneous magnetic moment of $\mathrm{Er}_{2} \mathrm{Fe}_{17}$ is expressed by subtracting the contribution of both sublattices, due to their antiparallel alignment: ${ }^{66}$

$$
\mu_{\mathrm{Er}_{2} \mathrm{Fe}_{17}}^{\text {Total }}=\mu_{\mathrm{Fe}}^{\text {Total }}-\mu_{\mathrm{Er}}^{\text {Total }}=\sum_{i=1}^{17} \mu_{\mathrm{Fe}_{i}}-\sum_{i=1}^{2} \mu_{\mathrm{Er}_{i}} .
$$

The magnetic moment at $T=2 \mathrm{~K}, \mu_{\mathrm{Er}_{2} \mathrm{Fe}_{17}}^{\mathrm{Total}}=14.7 \mu_{B}$, is in good agreement with the values obtained from the magnetic measurements $\left(\mu_{\mathrm{Er}_{2} \mathrm{Fe}_{17}}^{\mathrm{Total}}=16 \mu_{B}\right)$. The main feature of the curve is the broad maximum between 0.4 and $0.7 T_{C}$, which corresponds to the $120-210 \mathrm{~K}$ temperature range [see Fig. 5(b)]. Using a mean-field Hamiltonian including both crystal-field and exchange interactions (see Sec. III), we have calculated the temperature dependence of the magnetic moments for the $\mathrm{Er}^{3+}$ and $\mathrm{Fe}$ ions and their sublattices [see Figs. 5(a) and 5(b)]. It is worth noting that a remarkable agreement is observed, without the addition of any adjustable parameter. In particular, the smooth maximum of $\mu_{\mathrm{Er}_{2} \mathrm{Fe}_{17}}^{\mathrm{Total}_{17}}$ well described, and corresponds with the expected behavior for a ferrimagnetic system. Such behavior was predicted

TABLE I. Atomic coordinates for each crystallographic site obtained from the D2B neutron powder diffraction pattern collected at $T=320 \mathrm{~K}$ (reliable factors: $R_{p}=6.41, R_{\mathrm{wp}}=8.94, R_{\mathrm{exp}}=2.74$, Bragg $R$ factor $R_{B}=7.70$, and $R_{F}=6.06$ ) as well as the magnetic moments, obtained at $T=2 \mathrm{~K}$ (reliable factors: $R_{p}=4.92, R_{\mathrm{wp}}=7.53, R_{\exp }=0.27, R_{B}=4.70, R_{F}=2.70$, and magnetic $R$ factor $=6.14$ ) for the studied $\mathrm{Er}_{2} \mathrm{Fe}_{17}\left(P 6_{3} / \mathrm{mmc}\right)$ compound. Unit cell parameters and volume at $320 \mathrm{~K}$ as well as the total magnetic moment of each sublattice and of the magnetic unit cell at $2 \mathrm{~K}$ are shown.

\begin{tabular}{|c|c|c|c|c|c|}
\hline Atom site & $x$ & $y$ & $z$ & $B\left(\AA^{2}\right)$ & $\mu\left(\mu_{B}\right)$ \\
\hline $\operatorname{Er}(2 b)$ & 0.000 & 0.000 & 0.250 & $0.60(5)$ & $9.03(1)$ \\
\hline $\operatorname{Er}(2 d)$ & 0.333 & 0.667 & 0.750 & $0.60(5)$ & $9.03(1)$ \\
\hline $\mathrm{Fe}(4 f)$ & 0.333 & 0.667 & $0.106(1)$ & $0.45(5)$ & $2.23(1)$ \\
\hline $\mathrm{Fe}(6 \mathrm{~g})$ & 0.500 & 0.000 & 0.000 & $0.39(5)$ & $2.04(1)$ \\
\hline $\mathrm{Fe}(12 \mathrm{j})$ & $0.329(1)$ & $0.958(1)$ & 0.250 & $0.59(3)$ & $1.98(1)$ \\
\hline $\mathrm{Fe}(12 \mathrm{k})$ & $0.166(1)$ & $0.332(1)$ & $0.983(1)$ & $0.15(3)$ & $1.73(1)$ \\
\hline$a=b(\AA)$ & $8.451(1)$ & & & $\mu_{\mathrm{Er}}^{\text {Total }}\left(\mu_{B}\right)$ & 18.1(1) \\
\hline$c(\AA)$ & $8.264(1)$ & & & $\mu_{\mathrm{Fe}}^{\text {Total }}\left(\mu_{B}\right)$ & $32.8(2)$ \\
\hline$V\left(\AA^{3}\right)$ & $511.27(1)$ & & & $\mu_{\mathrm{Er}_{2} \mathrm{Fe}_{17}}^{\mathrm{Total}}\left(\mu_{B}\right)$ & $14.7(1)$ \\
\hline
\end{tabular}




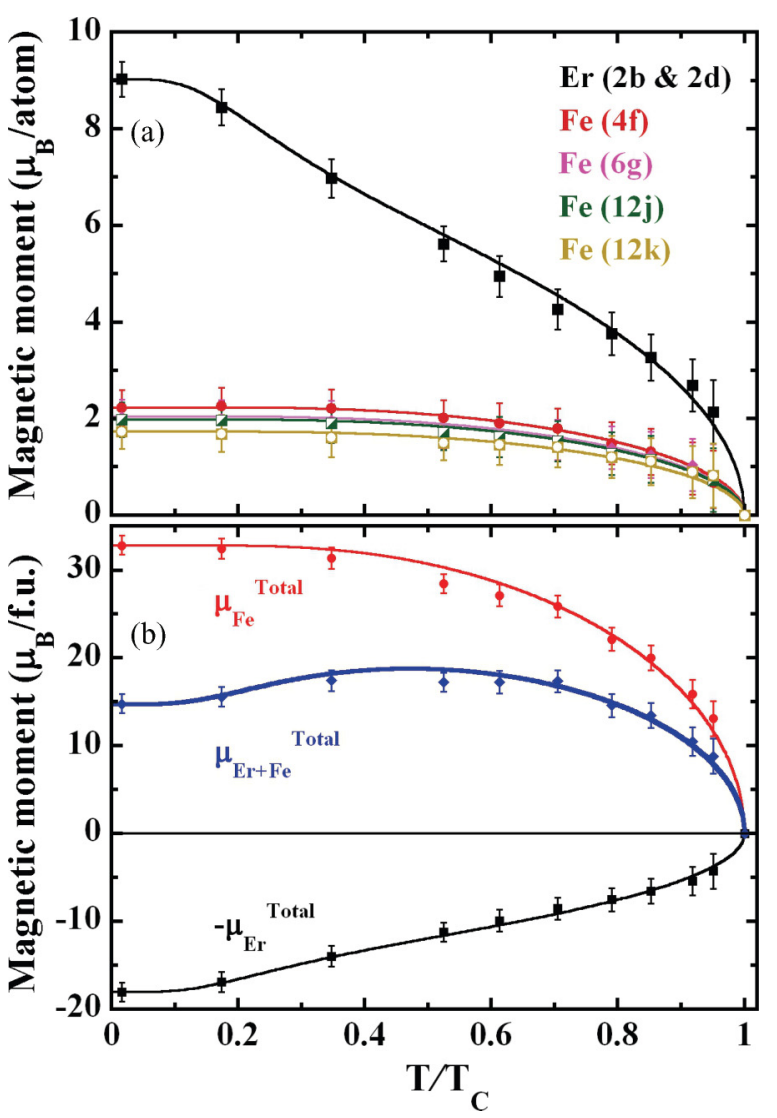

FIG. 5. (Color online) Reduced temperature dependence of the magnetic moment for (a) the two nonequivalent crystallographic Er sites and the four Fe sites and (b) similary for the Fe and Er-sublattices as well as for $\mathrm{Er}_{2} \mathrm{Fe}_{17}$. Solid lines represent the calculated temperature dependence of the magnetic moment (see text for more detail).

for the first time by Néel, ${ }^{67}$ and occurs in $\mathrm{Er}_{2} \mathrm{Fe}_{17}$ when $\mu_{\mathrm{Er}}^{\text {Total }}$ decreases more rapidly with temperature than $\mu_{\mathrm{Fe}}^{\text {Total }}$ does, and therefore the spontaneous magnetization increases. ${ }^{68}$ According to the Néel's theory of ferrimagnetism, the observed $M(T)$ behavior corresponds to that denoted as $\mathrm{P}$ type in Néel notation. ${ }^{67}$ The parameters that explain this behavior are $x=\frac{\mu_{\mathrm{Er}}}{\mu_{\mathrm{Fe}}}$ and $y=\frac{N_{\mathrm{Er}}}{N_{\mathrm{Fe}}}$, where $\mu_{\mathrm{Er}}\left(\mu_{\mathrm{Fe}}\right)$ and $N_{\mathrm{Er}}\left(N_{\mathrm{Fe}}\right)$ are the magnetic moments and the number of $\mathrm{Er}(\mathrm{Fe})$ atoms, respectively. In fact, when the product $x y$ is larger than 1 , the $M(T)$ curve differs from zero for $T \geqslant 0 \mathrm{~K}$, increases when the temperature is raised, passes through a maximum and, finally, drops sharply at $T_{C}$. This trend was experimentally found in $\mathrm{Er}_{2} \mathrm{Fe}_{17}$ [see Figs. 1 and 5(b)].

\section{Magnetovolume anomalies of $\mathrm{Er}_{2} \mathrm{Fe}_{17}$}

The temperature evolution of the lattice parameters and the unit cell volume (see Fig. 6) were obtained from the analysis of the diffraction patterns collected on D1B. $\mathrm{Er}_{2} \mathrm{Fe}_{17}$ exhibits several anomalies below $450 \mathrm{~K}$. On heating from $T=2 \mathrm{~K}$, we observe (i) a moderate decrease of the crystalline unit cell parameter along the $c$ axis up to $T \sim T_{C}$, while the basal-plane unit cell parameters slightly increase $(\sim 0.1 \%)$ in the same temperature range; (ii) an almost flat region between $T_{C}$ and 4/3 $T_{C}$, with very small variations of the cell parameters; (iii) both $a$ and $c$ cell parameters show a Grüneisen-like behavior when the temperature is greater than $450 \mathrm{~K}\left(1.5 T_{C}\right)$.

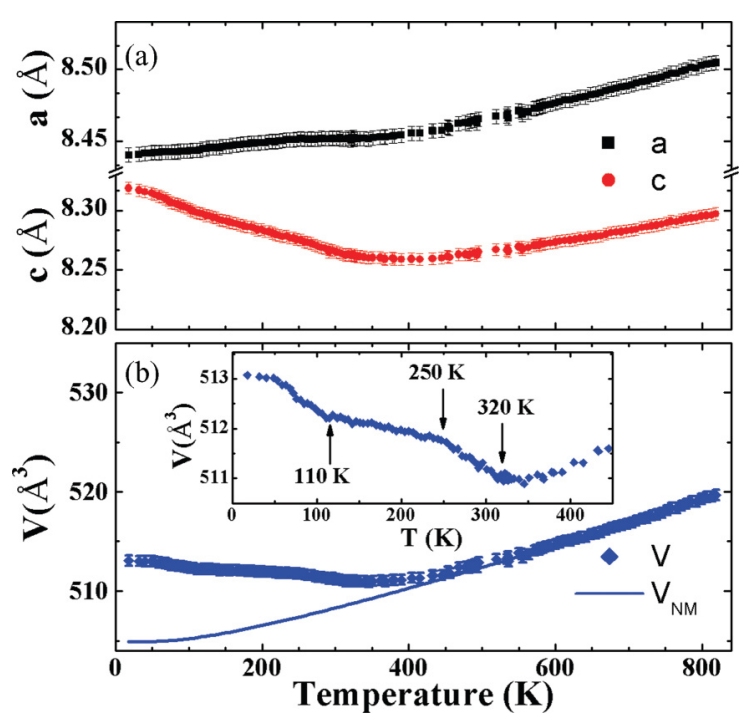

FIG. 6. (Color online) Temperature dependence of (a) the cell parameters and (b) the experimental unit cell volume $V(T)$ for $\mathrm{Er}_{2} \mathrm{Fe}_{17}$. The solid line $V_{\mathrm{NM}}$ corresponds to the nonmagnetic contribution to the unit cell volume, which is an extrapolation of $V(T)$ from the paramagnetic region down to low temperature using a Grüneisen law (see text for details).

In addition, $R_{2} \mathrm{Fe}_{17}$ intermetallic compounds are known to undergo large spontaneous magnetostrictive or magnetovolume deformations originated mainly from the Fe sublattice. ${ }^{21}$ This leads to Invar behavior within a wide temperature range. In fact, these magnetovolume effects are clearly illustrated in the temperature dependence of the cell volume, $V(T)$ [see Fig. 6(b) and the inset therein]. $V(T)$ is almost constant in the 0 to $60 \mathrm{~K}$ low-temperature region. On heating up to $110 \mathrm{~K}$ the volume clearly decreases (negative thermal expansion, $\mathrm{NTE}$ ). In the 110-250 K temperature range, $V(T)$ continues its decrease, but with a much lower slope. Further heating shows another region of NTE with a minimum value for the volume at $T \sim 320 \mathrm{~K}$. These anomalies in the unit cell volume were ascribed to the strong dependence of magnetic coupling with the $\mathrm{Fe}-\mathrm{Fe}$ distances, ${ }^{2,25,40,69}$ likewise many other Fe-based Invar alloys either in amorphous or crystalline state. ${ }^{59,70-72}$

At low temperatures, the unit cell volume is expanded respect to a nonmagnetic system [see Fig. 6(b), where both the experimental and the nonmagnetic cell volumes are plotted]. In order to estimate the nonmagnetic contribution to the unit cell volume using the Grüneisen relation, ${ }^{73-75}$ we have used the value of the bulk modulus $B=149 \mathrm{GPa}$ obtained from the refinement of the XRD patterns under high pressure (see below), whereas the value of the Debye temperature $\theta_{D} \sim 460 \mathrm{~K}$ and $\gamma \sim 350 \mathrm{meV}$ were extracted from the measured low-temperature heat capacity $\left(C_{P} \sim \gamma+\beta T^{2}\right){ }^{76}$ The Grüneisen parameter, $\Gamma=1.9$, and the nonmagnetic volume at zero temperature, $V_{0}=505 \AA^{3}$, were estimated from the fit.

The large volume spontaneous magnetostriction $\left(\omega_{S} \sim\right.$ $1.6 \times 10^{-2}$ at $T=5 \mathrm{~K}$ ) is the responsible for the difference between the experimental and the extrapolated values of the unit cell volume. The nonmagnetic unit cell volume 


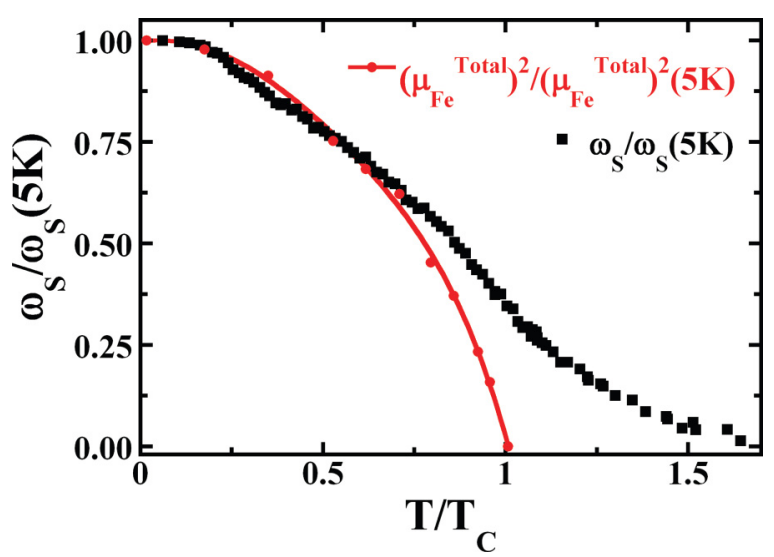

FIG. 7. (Color online) Reduced temperature dependence of the volume spontaneous magnetostriction, $\omega_{S}(T) / \omega_{S}$ at $5 \mathrm{~K}$, compared with that of the square of the magnetic moment over $\left(\mu_{\mathrm{Fe}}^{\mathrm{Total}}\right)^{2}(5 \mathrm{~K})$ for the Fe sublattice. The value of $\omega_{S}(5 \mathrm{~K})$ is $\sim 1.6 \times 10^{-2}$ and $\mu_{\mathrm{Fe}}^{\text {Total }}(5 \mathrm{~K})$ is $\sim 14.7 \mu_{B}$, respectively.

is smaller than the experimental one, which implies that the spontaneous magnetostriction $\omega_{S}(T)$ is negative below $450 \mathrm{~K}$. In other $R_{2} \mathrm{Fe}_{17}$ compounds, $\omega_{S}$ has been correlated with the square of the total Fe-sublattice magnetic moment, $\mu_{\mathrm{Fe}}^{2}{ }^{26,77}$ Figure 7 shows the trends of $\omega_{S}(T) / \omega_{S}(5 \mathrm{~K})$ and $\left(\mu_{\mathrm{Fe}}^{\text {Total }}\right)^{2}(T) /\left(\mu_{\mathrm{Fe}}^{\text {Total }}\right)^{2}(5 \mathrm{~K})$ as a function of the reduced temperature $T / T_{C}$. Both curves roughly overlap up to $T \sim$ $0.8 T_{C}$. At higher temperatures, the $\omega_{S}(T)$ curve deviates from $\left(\mu_{\mathrm{Fe}}^{\text {Total }}\right)^{2}(T)$, and at $T_{C}, \omega_{S}(T) / \omega_{S}(5 \mathrm{~K}) \sim 0.35$, suggesting the presence of short-range magnetic correlations, ${ }^{78}$ which disappear for $T>450 \mathrm{~K}\left(T / T_{C} \sim 1.5\right)$.

In addition, we have investigated the evolution of the unit cell crystal parameters and volume under hydrostatic pressure [see Figs. 8(a) and 8(b), where XRD patterns under $P=0$ and $15 \mathrm{GPa}$ are shown, respectively]. The shape of the Bragg diffraction peaks is flatter and the intensity decreases when the pressure increases, due to the deformation of the crystalline domains with increasing pressure. The XRD patterns were analyzed using the Le Bail method due to the extreme difficulty of performing a Rietveld analysis. A continuous shift of the diffraction peaks to higher $2 \theta$ angles with increasing pressure is consistent with a reduction of the unit cell parameters. The normalized unit cell parameters of $\mathrm{Er}_{2} \mathrm{Fe}_{17}$ as a function of the applied pressure are also shown in the inset of Fig. 8(c). A continuous decrease in both ratios is clearly observed, but the crystal structure does not change under pressures up to $P=15 \mathrm{GPa}$. The lattice contraction along the $c$ axis as well as that in the basal plane seems to be isotropic, since both curves almost match each other. Data corresponding to the unit cell volume versus applied pressure were fitted to the BirchMurnaghan equation of state. ${ }^{79}$ A value of $B=149 \pm 4 \mathrm{GPa}$ was obtained for the bulk modulus, which is consistent with those reported in the literature. ${ }^{55}$

\section{E. Magnetocaloric Effect of $\mathrm{Er}_{2} \mathrm{Fe}_{17}$}

Accurate values for the MCE were obtained from both magnetization and heat-capacity data. Hence the isothermal magnetic entropy change was estimated from two methods: from the $M(H)$ curves (see Fig. 2) together with Eq. (1), and

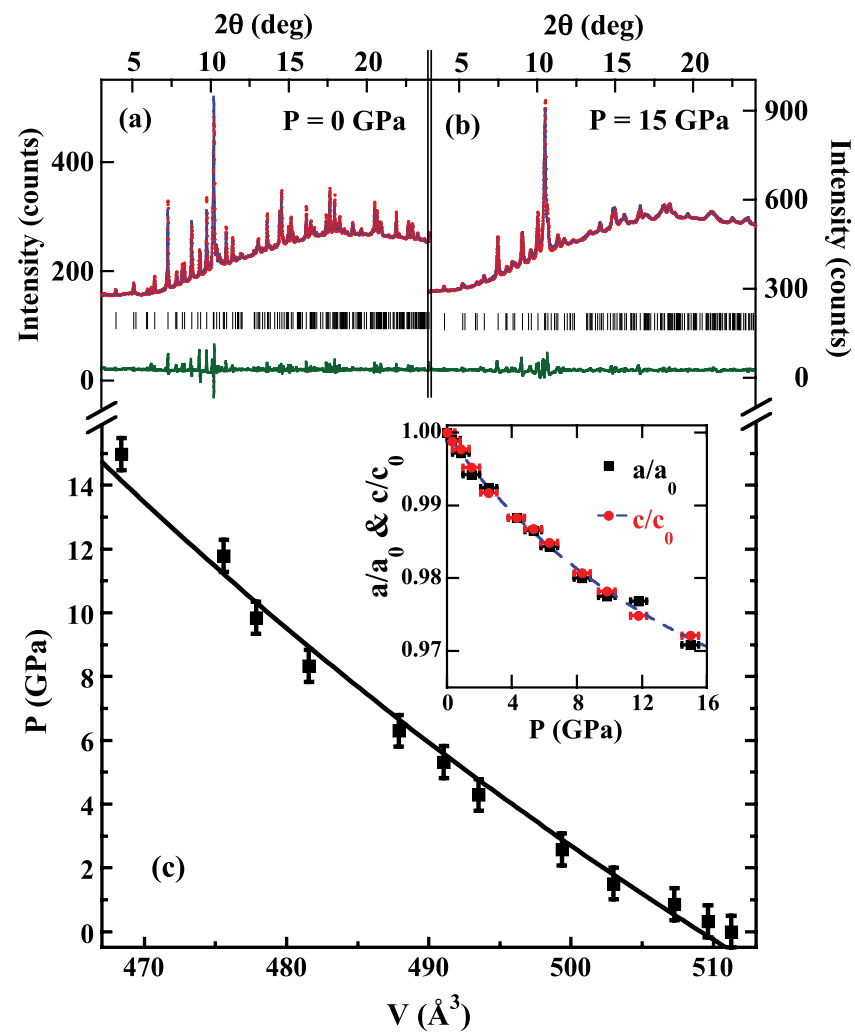

FIG. 8. (Color online) Observed (dots) and calculated (solid line) x-ray powder diffraction patterns for $\mathrm{Er}_{2} \mathrm{Fe}_{17}$ collected at (a) $P=0$ and (b) $15 \mathrm{GPa}$. The observed-calculated difference is depicted at the bottom of each figure. (c) Fit of the $P(V)$ curve using the BirchMurnaghan equation of state. (Inset) Pressure dependence of the unit cell parameters normalized to the value at $P=0 \mathrm{GPa}\left(a_{0}\right.$ and $\left.c_{0}\right)$ for the $\mathrm{Th}_{2} \mathrm{Ni}_{17}$-type crystal structure in $\mathrm{Er}_{2} \mathrm{Fe}_{17}$ at room temperature. The dashed line is a guide for the eyes.

from the heat-capacity data measured under several applied magnetic field values (see Fig. 3) and Eq. (2). The $\Delta S_{M}(T)$ curves for the $\mathrm{Er}_{2} \mathrm{Fe}_{17}$ alloy are plotted in the upper part of Fig. 9. Peaks located at $T=300 \mathrm{~K}$ correspond to a direct MCE associated with the magnetic transition from the ferrimagnetic to paramagnetic state. The magnitude of $\Delta S_{M}(T)$ obtained from $M(H)$ data at $300 \mathrm{~K}$ under $\Delta H=$ $50 \mathrm{kOe}$ is $-3.6 \mathrm{~J}(\mathrm{kgK})^{-1}$, which is quite close to the value reported in Ref. 80, while at $\Delta H=80 \mathrm{kOe}, \Delta S_{M}(T)$ reaches a value of $-4.7 \mathrm{~J}(\mathrm{kgK})^{-1} \cdot \mathrm{Er}_{2} \mathrm{Fe}_{17}$ also exhibits an inverse MCE with a maximum value of $\Delta S_{M}=1.3 \mathrm{~J}(\mathrm{kgK})^{-1}(\Delta H=$ $80 \mathrm{kOe})$ at $T \sim 40 \mathrm{~K}$. These values for the inverse and direct MCE are in good agreement with those found from heatcapacity measurements [see Fig. 10(a)], where $\Delta S_{M}(T)=$ $-5.2(1.2) \mathrm{J}(\mathrm{kgK})^{-1}$ for the direct (inverse) $\mathrm{MCE}$ at $300(40) \mathrm{K}$, respectively, under a magnetic field change of $80 \mathrm{kOe}$. Another noticeable result is that $\Delta S_{M}$ vanishes in the crossover from the inverse to the direct $\mathrm{MCE}$ at around $150 \mathrm{~K}$. At this temperature, the $V(T)$ curve exhibits a NTE behavior, while the total magnetic moment displays a broad maximum.

The existence of an inverse MCE has been theoretically predicted in the framework of the ferrimagnetic character of the magnetic structure. ${ }^{68}$ However, in this work, we have studied the effects of the particular CEF level scheme of $\mathrm{Er}^{3+}$ ion 


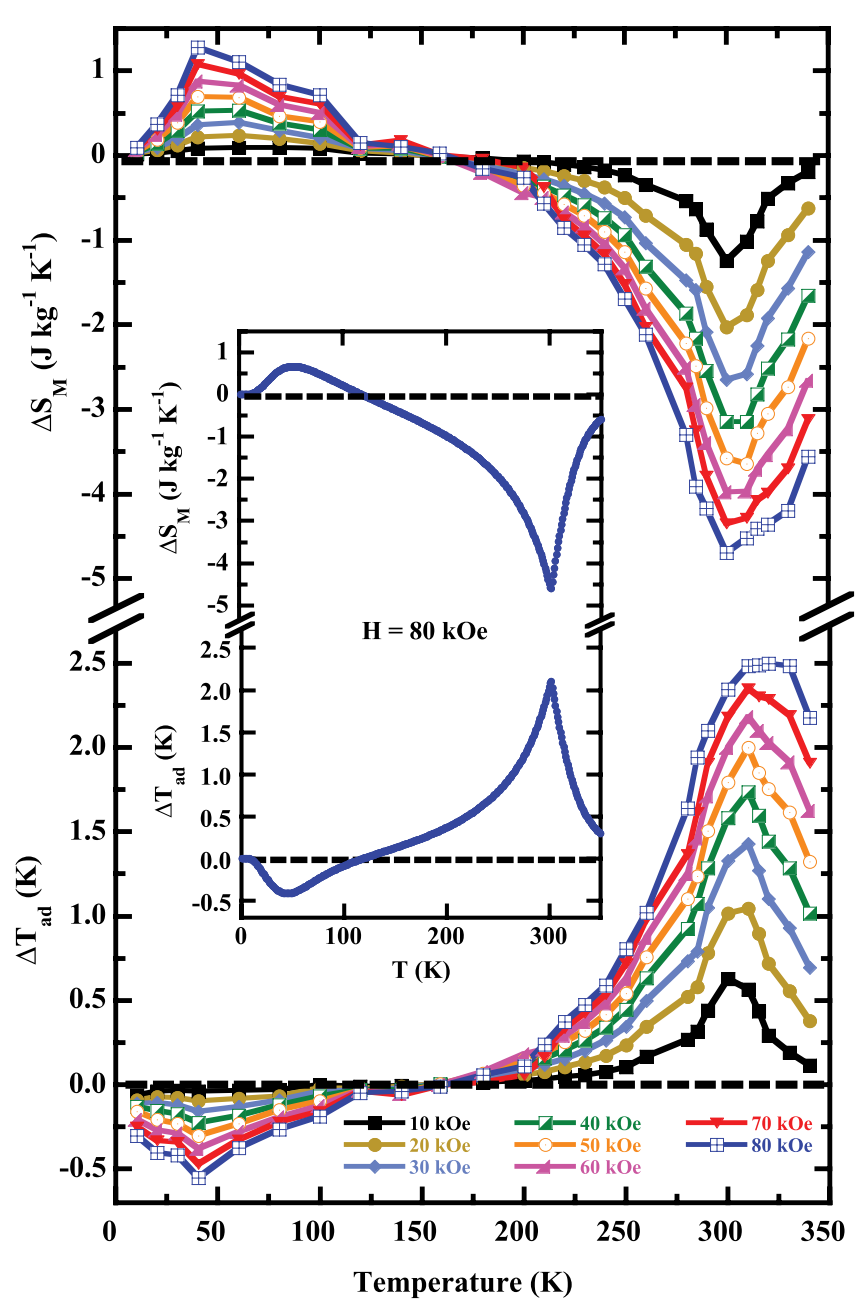

FIG. 9. (Color online) Temperature dependence of (a) the isothermal magnetic entropy and (b) the adiabatic temperature changes under $\Delta H$ up to $80 \mathrm{kOe}$ for $\mathrm{Er}_{2} \mathrm{Fe}_{17}$ determined from magnetization measurements. The insets show the theoretical values of $\Delta S_{M}$ and $\Delta T_{\text {ad }}$ using the CEF and exchange parameters quoted in Sec. III (see text).

on the MCE. ${ }^{10}$ The inset of Fig. 9(a) shows the temperature dependence of the magnetic entropy change considering the contributions of the $\mathrm{Fe}$ sublattice due to Heisenberg exchange interactions and those of the CEF and Heisenberg exchange interactions for the Hamiltonian related to the $\mathrm{Er}$ sublattice (see Sec. III). The direct and inverse magnetocaloric effects are qualitatively well accounted by the theoretical model, even though the crossover where the MCE is zero is predicted to be $\sim 120 \mathrm{~K}$, while the experimental determination is found at $150 \mathrm{~K}$.

Taking into account Eqs. (2) and (3), the adiabatic temperature change was estimated from the specific heat at zero-field and the magnetic entropy change obtained from both the $M(H)$ curves [see Fig. 9(b)] as well as the heat-capacity data [see Fig. 10(b)]. The values of $\Delta T_{\mathrm{ad}}$ at 300(40) $\mathrm{K}$ for the direct (inverse) MCE are 2.5(-0.6) and 3.2(-0.6) $\mathrm{K}$ for the determination obtained from magnetization and heatcapacity data, respectively. These main features exhibited by the experimental $\Delta T_{\text {ad }}$ curves also emerge in the theoretical

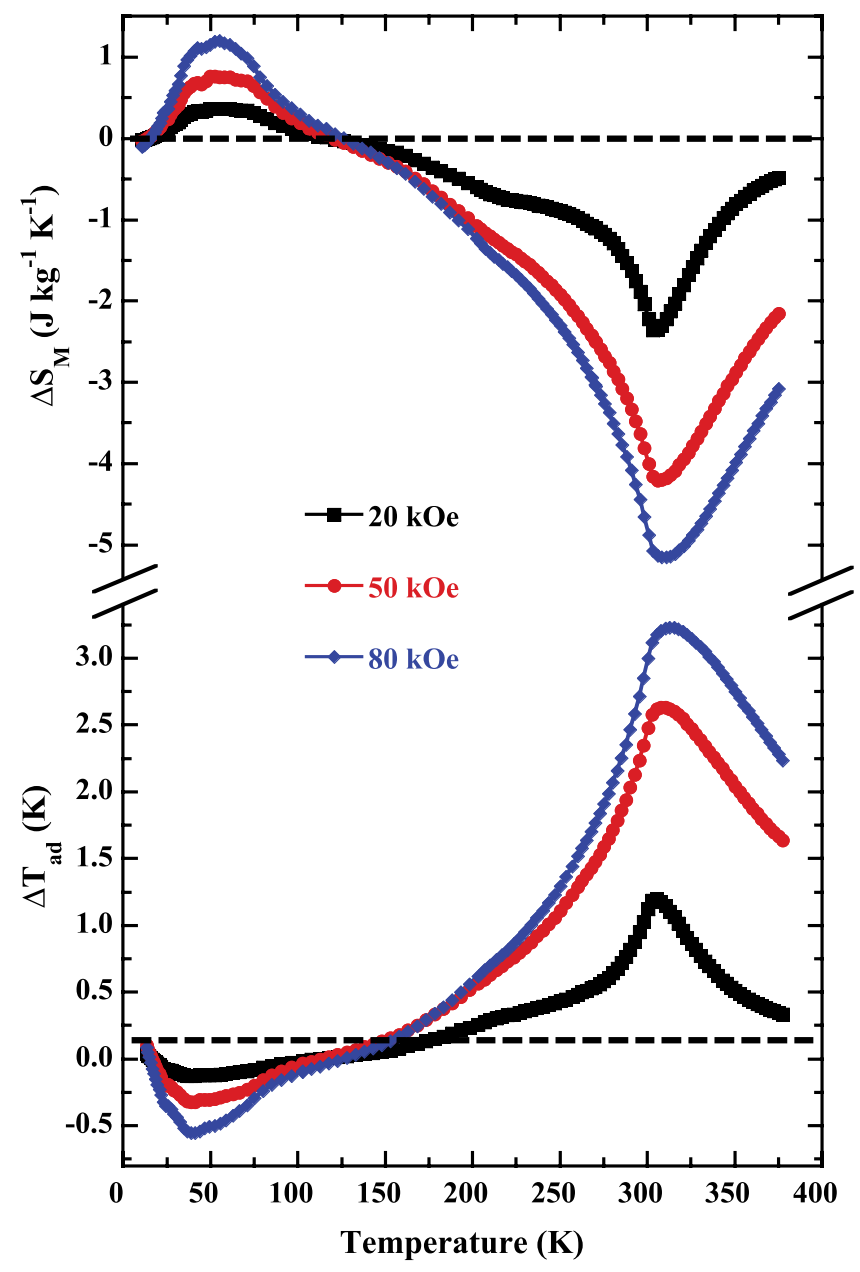

FIG. 10. (Color online) Temperature dependence of (a) the isothermal magnetic entropy and (b) the adiabatic temperature changes under $\Delta H$ of 20,50 , and $80 \mathrm{kOe}$ for $\mathrm{Er}_{2} \mathrm{Fe}_{17}$ determined from heat-capacity measurements (see text).

calculation [see inset in Fig. 9(b)]. Furthermore, calculated and experimental values are reasonably close, even though the shape of the theoretical peaks looks narrower, probably due to finite grain sizes. The discrepancies between the experimental results and the model in both adiabatic temperature change and magnetic entropy change suggest that other interactions must be taken into account to describe more accurately the experimental behavior. The magnetoelastic coupling is the main candidate, in so far as the unit cell volume also has anomalous behavior below $T_{C}$. However, further investigations are needed to elucidate these discrepancies, as the implementation of such interaction is labor intensive since the elastic and magnetoelastic constants must be known.

\section{CONCLUSIONS}

In summary, $\mathrm{Er}_{2} \mathrm{Fe}_{17}$ exhibits noticeable magnetovolume anomalies below the Curie point, evidenced by different changes of slope in the temperature dependence of the unit cell volume (the $c$ crystallographic cell parameter unceasingly diminishes with increasing temperature, while the basalplane cell parameters slightly increase, being almost flat 
around $T_{C}$ ). These magnetovolume anomalies give rise to a positive spontaneous magnetostriction with a maximum $\left(\omega_{S} \sim 1.6 \times 10^{-2}\right)$ at $T=5 \mathrm{~K}$ that falls down to zero well above the Curie temperature (owing to the existence of short-range ordering effects). The existence of both direct $\left[\Delta S_{M} \sim-4.7 \mathrm{~J}(\mathrm{kgK})^{-1}\right.$ and $\Delta T_{\mathrm{ad}} \sim 2.5 \mathrm{~K}$ for $80 \mathrm{kOe}$ ] and inverse $\left[\Delta S_{M} \sim 1.3 \mathrm{~J}(\mathrm{kgK})^{-1}\right.$ and $\Delta T_{\mathrm{ad}} \sim-0.6 \mathrm{~K}$ for $80 \mathrm{kOe}]$ magnetocaloric effects determined from magnetization measurements are qualitatively well described considering a mean field Hamiltonian that includes both crystalline electric field and exchange interactions. The model also predicts that as we increase the temperature, the sign of $\Delta S_{M}$ and $\Delta T_{\mathrm{ad}}$ changes at a certain temperature where the crossover from inverse to direct magnetocaloric effect occurs.

\section{ACKNOWLEDGMENTS}

Financial support from Spanish MICINN (MAT201127573-C04-02) and from the Basque Government (IT-34707) is acknowledged. J.L.S.Ll. acknowledges the support received from CONACYT, Mexico, under the project CB2010-01-156932, and Laboratorio Nacional de Investigaciones en Nanociencias y Nanotecnología (LINAN, IPICyT). J.A.R.V. acknowledges the support from the research project MAT2007-61621. We thank ILL and CRG-D1B for allocating neutron beamtime, and ESRF for synchrotron beamtime. The SCTs at the University of Oviedo and the technical support received from M.Sc. G. J. Labrada-Delgado and B. A. Rivera-Escoto (DMA, IPICyT) are also acknowledged. *alvarezpablo.uo@uniovi.es

†pgorria@uniovi.es

${ }^{\ddagger}$ jabr@uniovi.es

${ }^{1}$ D. Haskel, Y. B. Lee, B. N. Harmon, Z. Islam, J. C. Lang, G. Srajer,

Y. Mudryk, K. A. Gschneidner, and V. K. Pecharsky, Phys. Rev. Lett. 98, 247205 (2007).

${ }^{2}$ V. Hari Babu, G. Markandeyulu, and A. Subrahmanyam, Appl. Phys. Lett. 90, 252513 (2007).

${ }^{3}$ S. B. Roy, P. Chaddah, V. K. Pecharsky, and K. A. Gschneidner Jr., Acta Mater. 56, 5895 (2008)

${ }^{4}$ S. N. Jammalamadaka, N. Mohapatra, S. D. Das, and E. V. Sampathkumaran, Phys. Rev. B 79, 060403(R) (2009).

${ }^{5}$ E. Brück, J. Phys. D: Appl. Phys. 38, R381 (2005).

${ }^{6}$ O. Gutfleisch, M. A. Willard, E. Brück, C. H. Chen, S. G. Sankar, and J. P. Liu, Adv. Mater. 23, 821 (2011).

${ }^{7}$ O. Tegus, E. Brück, K. H. J. Buschow, and F. R. de Boer, Nature (London) 415, 150 (2002).

${ }^{8}$ A. Yan, K. H. Müller, and O. Gutfleisch, J. Appl. Phys. 97, 036102 (2005).

${ }^{9}$ P. Álvarez, J. Sánchez-Marcos, P. Gorria, L. Fernández Barquín, and J. A. Blanco, J. Alloys Compd. 504, S150 (2010).

${ }^{10}$ P. Álvarez, P. Gorria, and J. A. Blanco, Phys. Rev. B 84, 024412 (2011).

${ }^{11}$ A. Rowe and A. Tura, Int. J. Refrig. 29, 1286 (2006).

${ }^{12}$ P. Álvarez, J. L. Sánchez Llamazares, P. Gorria, and J. A. Blanco, Appl. Phys. Lett. 99, 232501 (2011).

${ }^{13}$ K. H. J. Buschow, Rep. Prog. Phys. 40, 1179 (1977).

${ }^{14}$ K. A. Gschneidner Jr, V. K. Pecharsky, and A. O. Tsokol, Rep. Prog. Phys. 68, 1479 (2005).

${ }^{15}$ P. Gorria, J. L. Sánchez Llamazares, P. Álvarez, M. J. Pérez, J. Sánchez Marcos, and J. A. Blanco, J. Phys. D: Appl. Phys. 41, 192003 (2008).

${ }^{16}$ J. L. Wang, S. J. Campbell, O. Tegus, C. Marquina, and M. R. Ibarra, Phys. Rev. B 75, 174423 (2007).

${ }^{17}$ A. Kreyssig, S. Chang, Y. Janssen, J. W. Kim, S. Nandi, J. Q. Yan, L. Tan, R. J. McQueeney, P. C. Canfield, and A. I. Goldman, Phys. Rev. B 76, 054421 (2007).

${ }^{18}$ R. Boada, C. Piquer, M. A. Laguna-Marco, and J. Chaboy, Phys. Rev. B 82, 052407 (2010).

${ }^{19}$ M. Palit, J. A. Chelvane, H. Basumatary, S. Banumathy, A. K. Singh, S. Pandian, and V. Chandrasekaran, Intermetallics 18, 1027 (2010).
${ }^{20}$ Binary Alloy Phase Diagrams, edited by T. B. Massalski, 2nd ed. (ASM International, Materials Park, OH, 1990).

${ }^{21}$ D. Givord, R. Lemaire, W. James, J. M. Moreau, and J. Shah, IEEE Trans. Magn. 7, 657 (1971).

${ }^{22}$ D. Givord and R. Lemaire, IEEE Trans. Magn. 10, 109 (1974).

${ }^{23}$ J. B. A. A. Elemans and K. H. J. Buschow, Phys. Status Solidi A 24, K125 (1974).

${ }^{24}$ Y. Janssen, S. Chang, A. Kreyssig, A. Kracher, Y. Mozharivskyj, S. Misra, and P. C. Canfield, Phys. Rev. B 76, 054420 (2007).

${ }^{25}$ P. Gorria, P. Álvarez, J. Sánchez-Marcos, J. L. Sánchez-Llamazares, M. J. Pérez, and J. A. Blanco, Acta Mater. 57, 1724 (2009).

${ }^{26}$ A. V. Andreev, F. R. de Boer, T. H. Jacobs, and K. H. J. Buschow, Physica B 175, 361 (1991).

${ }^{27}$ G. J. Long, G. K. Marasinghe, S. Mishra, O. A. Pringle, Z. Hu, W. B. Yelon, D. P. Middleton, K. H. J. Buschow, and F. Grandjean, J. Appl. Phys. 76, 5383 (1994).

${ }^{28}$ J. M. D. Coey, J. Magn. Magn. Mater. 248, 441 (2002).

${ }^{29}$ F. Grandjean, O. Isnard, D. Hautot, and G. J. Long, Phys. Rev. B 63, 014406 (2000).

${ }^{30}$ K. Mandal, A. Yan, P. Kerschl, A. Handstein, O. Gutfleisch, and K. Muller, J. Phys. D: Appl. Phys. 37, 2628 (2004).

${ }^{31}$ Y. V. Knyazev, A. V. Lukoyanov, Y. I. Kuz'min, A. G. Kuchin, and I. A. Nekrasov, Phys. Rev. B 73, 094410 (2006).

${ }^{32}$ F. Canepa, S. Cirafici, M. Napoletano, and R. Masini, Phys. Rev. B 76, 104402 (2007).

${ }^{33}$ M. D. Kuz'min, Y. Skourski, K. P. Skokov, K. H. Müller, and O. Gutfleisch, Phys. Rev. B 77, 132411 (2008).

${ }^{34}$ P. Álvarez, P. Gorria, V. Franco, J. Sánchez Marcos, M. J. Pérez, J. L. Sánchez Llamazares, I. Puente Orench, and J. A. Blanco, J. Phys.: Condes. Matter 22, 216005 (2010).

${ }^{35}$ Y. Li, R. G. Graham, D. S. Bunbury, P. W. Mitchell, and M. A. H. McCausland, J. Magn. Magn. Mater. 140-144, 1007 (1995).

${ }^{36}$ A. V. Lukoyanov, E. E. Kokorina, M. V. Medvedev, and I. A. Nekrasov, Phys. Rev. B 80, 104409 (2009).

${ }^{37}$ R. Kumar and W. B. Yelon, J. Appl. Phys. 67, 4641 (1990).

${ }^{38}$ I. S. Tereshina, S. A. Nikitin, J. Stepien-Damm, L. D. Gulay, N. Y. Pankratov, A. A. Salamova, V. N. Verbetsky, and W. Suski, J. Alloys Compd. 329, 31 (2001).

${ }^{39}$ I. Tereshina, S. Nikitin, W. Suski, J. Stepien-Damm, W. Iwasieczko, H. Drulis, and K. Skokov, J. Alloys Compd. 404-406, 172 (2005). 
${ }^{40}$ N. Yang, K. W. Dennis, R. W. McCallum, M. J. Kramer, Y. Zhang, and P. L. Lee, J. Magn. Magn. Mater. 311, 630 (2007).

${ }^{41}$ P. Alvarez, J. L. Sanchez-Llamazares, M. J. Perez, B. Hernando, J. D. Santos, J. Sanchez Marcos, J. A. Blanco, and P. Gorria, J. Non-Cryst. Solids 354, 5172 (2008).

${ }^{42}$ J. L. Sánchez Llamazares, M. J. Pérez, P. Álvarez, J. D. Santos, M. L. Sánchez, B. Hernando, J. A. Blanco, J. Sánchez-Marcos, and P. Gorria, J. Alloys Compd. 483, 682 (2009).

${ }^{43}$ P. Alvarez, P. Gorria, J. Sánchez-Llamazares, M. J. Pérez, V. Franco, M. Reiffers, J. Kovac, I. Puente-Orench, and J. A. Blanco, Mat. Chem. Phys. 131, 18 (2011).

${ }^{44}$ P. Alvarez, P. Gorria, J. L. Sánchez-Llamazares, M. J. Pérez, V. Franco, M. Reiffers, I. Curlik, E. Gazo, J. Kovac, and J. A. Blanco, Intermetallics 19, 982 (2011).

${ }^{45}$ K. A. Gschneidner Jr, J. Alloys Compd. 193, 1 (1993).

${ }^{46}$ A. P. Hammersley, S. O. Svensson, M. Hanfland, A. N. Fitch, and D. Hausermann, High Press. Res. 14, 235 (1996).

${ }^{47}$ J. Rodríguez-Carvajal, Physica B 192, 55 (1993).

${ }^{48}$ A. R. Dinesen, S. Linderoth, and S. Morup, J. Phys.: Condes. Matter 17, 6257 (2005).

${ }^{49}$ N. de Oliveira and P. von Ranke, Phys. Rep. 489, 89 (2010).

${ }^{50}$ M. T. Hutchings, in Solid State Physics, edited by F. Seitz and D. Turnbull, Vol. 16 (Academic Press, New York, 1964).

${ }^{51}$ K. Clausen and B. Lebech, J. Phys. C: Solid State Phys. 15, 5095 (1982).

${ }^{52}$ J. A. Blanco, D. Gignoux, J. C. Gómez Sal, and D. Schmitt, J. Magn. Magn. Mater. 104-107, 1273 (1992).

${ }^{53}$ A. V. Andreev, F. R. de Boer, T. H. Jacobs, and K. H. J. Buschow, J. Magn. Magn. Mater. 104-107, 1305 (1992).

${ }^{54}$ J. L. Wang, M. R. Ibarra, C. Marquina, B. García-Landa, W. X. Li, N. Tang, W. Q. Wang, F. M. Yang, and G. H. Wu, J. Appl. Phys. 92, 1453 (2002).

${ }^{55}$ M. Brouha, K. Buschow, and A. Miedema, IEEE Trans. Magn. 10, 182 (1974).

${ }^{56}$ J. Kamarad, O. Mikulina, Z. Arnold, B. Garcia-Landa, and M. R. Ibarra, J. Appl. Phys. 85, 4874 (1999).

${ }^{57}$ B. D. Cullity, Introduction to Magnetic Materials (Addison-Wesley, Reading, MA, 1972).

${ }^{58}$ M. D. Kuz'min, Y. Skourski, K. P. Skokov, and K. H. Müller, Phys. Rev. B 75, 184439 (2007).

${ }^{59}$ P. Gorria, D. Martinez-Blanco, M. J. Perez, J. A. Blanco, A. Hernando, M. A. Laguna-Marco, D. Haskel, N. SouzaNeto, R. I. Smith, W. G. Marshall, G. Garbarino, M. Mezouar, A. Fernandez-Martinez, J. Chaboy, L. Fernandez Barquin, J. A. Rodriguez Castrillon, M. Moldovan, J. I. Garcia Alonso, J. Zhang, A. Llobet, and J. S. Jiang, Phys. Rev. B 80, 064421 (2009).

${ }^{60}$ J. A. Blanco, D. Gignoux, and D. Schmitt, Z. Phys. B 89, 343 (1992).
${ }^{61}$ O. Isnard, S. Miraglia, C. Kolbeck, E. Tomey, J. L. Soubeyroux, D. Fruchart, M. Guillot, and C. Rillo, J. Alloys Compd. 178, 15 (1992).

${ }^{62}$ International Tables for Crystallography, edited by T. Hahn, H. Fuess, T. Hahn, H. Wondratschek, U. Müller, U. Shmueli, E. Prince, A. Authier, V. Kopský, D. B. Litvin, M. G. Rossmann, E. Arnold, S. Hall, and B. McMahon, 1st ed., Vol. A (International Union of Crystallography, Chester, England, 2006).

${ }^{63}$ A. N. Christensen and R. G. Hazell, Acta Chem. Scand. A: Phys. Inorg. Chem. 34, 455 (1980).

${ }^{64}$ V. I. Voronin, I. F. Berger, A. G. Kuchin, D. V. Sheptyakov, and A. M. Balagurov, J. Alloys Compd. 315, 82 (2001).

${ }^{65}$ C. Piqué, J. A. Blanco, R. Burriel, E. Abad, M. Artigas, and M. T. Fernández-Díaz, Phys. Rev. B 75, 224424 (2007).

${ }^{66}$ Z. H. Cheng, B. G. Shen, Q. W. Yan, H. Q. Guo, D. F. Chen, C. Gou, K. Sun, F. R. de Boer, and K. H. J. Buschow, Phys. Rev. B 57, 14299 (1998).

${ }^{67}$ L. Néel, Ann. Phys. Paris 3, 137 (1948).

${ }^{68}$ P. J. von Ranke, N. A. de Oliveira, B. P. Alho, E. J. R. Plaza, V. S. R. de Sousa, L. Caron, and M. S. Reis, J. Phys.: Condes. Matter 21, 056004 (2009).

${ }^{69}$ E. Girt, Z. Altounian, I. P. Swainson, K. M. Krishnan, and G. Thomas, J. Appl. Phys. 85, 4669 (1998).

${ }^{70}$ J. M. Barandiaran, P. Gorria, I. Orue, M. L. Fdez-Gubieda, F. Plazaola, and A. Hernando, Phys. Rev. B 54, 3026 (1996).

${ }^{71}$ P. Gorria, D. Martinez-Blanco, J. A. Blanco, A. Hernando, J. S. Garitaonandia, L. Fernandez Barquin, J. Campo, and R. I. Smith, Phys. Rev. B 69, 214421 (2004).

${ }^{72}$ A. Martinez, J. Spottorno, A. I. Figueroa, F. Bartolome, L. M. Garcia, C. Prestipino, A. Hernando, and P. Crespo, Phys. Rev. B 82, 012406 (2010)

${ }^{73}$ A. V. Andreev and S. Danis, J. Magn. Magn. Mater. 320, E168 (2008).

${ }^{74}$ A. Lindbaum and M. Rotter, in Handbook of Magnetic Materials, edited by K. H. J. Buschow, Vol. 14 (North Holland, Amsterdam, 2002), Chap. 4, p. 307.

${ }^{75}$ O. Söderberg, A. Sozinov, Y. Ge, S.-P. Hannula, and V. Lindroos, in Handbook of Magnetic Materials, edited by K. H. J. Buschow, Vol. 16 (North Holland, Amsterdam, 2006), Chap. 1, p. 1.

${ }^{76}$ J. A. Blanco, M. de Podesta, J. I. Espeso, J. C. Gómez Sal, C. Lester, K. A. McEwen, N. Patrikios, and J. Rodríguez Fernández, Phys. Rev. B 49, 15126 (1994).

${ }^{77}$ A. V. Andreev and A. Lindbaum, J. Alloys Compd. 297, 43 (2000).

${ }^{78}$ E. A. Tereshina and A. V. Andreev, Intermetallics 18, 1205 (2010).

${ }^{79}$ H. Zhang, X. Wu, K. G. Nickel, J. Chen, and V. Presser, J. Appl. Phys. 106, 013519 (2009).

${ }^{80}$ H. Chen, Y. Zhang, J. Han, H. Du, C. Wang, and Y. Yang, J. Magn. Magn. Mater. 320, 1382 (2007). 